\title{
UMA ANÁLISE DA INFLUÊNCIA DA ESTOCASTICIDADE DAS INFORMAÇÕES SOBRE UM MODELO DE PROGRAMAÇÃO LINEAR
}

\author{
Neida Maria Patias Volpi* \\ Celso Carnieri* \\ Carlos Roberto Sanquetta** \\ *Depto. de Matemática, UFPR, \\ **Depto. de Silvicultura e Manejo, UFPR
}

\begin{abstract}
Resumo
Neste trabalho foi discutido o impacto de perturbações estocásticas em um modelo de planejamento florestal. Foi desenvolvido um modelo de programação linear e uma abordagem, através de simulações estocásticas, para analisar e quantificar a variabilidade que ocorre nos valores da função objetivo, perante a natureza estocástica dos dados que alimentam o modelo. Um programa para efetuar tais simulações foi desenvolvido. O conceito de razão de variabilidade objeto-sistema foi introduzido para medir a suscetibilidade do sistema em relação às variações ocorridas no objeto.
\end{abstract}

Palavras-chave: planejamento florestal, programação linear, estocasticidade.

\begin{abstract}
The impact of stochastic perturbations in a forest planning model was discussed in this work. It was developed a linear programming and an approach, through stochastic simulations, to analyse and quantify the variability that occurs in the values of the objective function, because of the nature of the data that are used in the model. A program to do such simulations was developed. A new concept was introduced, called object-system ratio of variability, in order to measure the sensitivity of the response of the system when variations occur in the object.
\end{abstract}

Keywords: forest planning, linear programming, stochasticity. 


\section{INTRODUÇÃO}

O principal objetivo deste trabalho é analisar e quantificar o impacto que informações estocásticas que alimentam um modelo de programação linear, produzem sobre o valor da função objetivo. O modelo utilizado foi de planejamento florestal.

Empresas florestais possuem áreas plantadas com espécies florestais adequadas à sua atuação no mercado, seja madeira para serraria, polpa celulósica, madeira podada e outros.

Define-se estrato florestal como uma certa área de plantio com homogeneidade de espécie, mesma idade de plantio, igual distância ao centro de operações (para onde é transportada a madeira) e mesmo índice de sítio (que define a qualidade da terra).

Um regime de manejo significa um conjunto de decisões ou atividades, que são implementadas num determinado estrato florestal (por exemplo: podas, desbastes e corte final em idades prédefinidas).

O modelo de planejamento florestal considerado, procura o melhor regime de manejo para cada estrato florestal, de forma a maximizar a renda líquida da empresa. Além das decisões relativas aos manejos que devem ser considerados em cada estrato, visando obter os produtos florestais da empresa, outras variáveis podem ser consideradas no planejamento, tais como compras e vendas de madeira ou de determinados produtos, em caso de necessidade.

As atividades florestais, como as de manejo, são muito complexas e particulares, pois envolvem um produto que tem origem biológica e rotação de longo espectro. O mercado a longo prazo pode alterar consideravelmente preços e demandas dos produtos florestais. Os custos inerentes ao manejo também são suscetíveis a modificações. Planejamento é uma questão fundamental para as empresas florestais, porém, em si não tem valor se os dados que alimentam tais modelos não representam a realidade.

É comum o uso de modelos determinísticos de programação matemática, para auxiliar no planejamento de uma empresa florestal, porém a área florestal tem a particularidade de que várias das suas informações são amostrais, obedecendo equações da forma $\boldsymbol{y}_{\boldsymbol{i}}=\boldsymbol{\mu}+\boldsymbol{\varepsilon}_{\boldsymbol{i}}, \boldsymbol{i}=1,2, \ldots \boldsymbol{n}$, onde $\boldsymbol{\mu}$ é a parte sistemática que é avaliada de fato e $\boldsymbol{\varepsilon}_{\boldsymbol{i}}$ é a componente estocástica, que entra como uma fonte de perturbação. Tomam-se decisões usandose resultados de um modelo de programação linear determinístico, alimentado por $\boldsymbol{y}_{\boldsymbol{i}}=\boldsymbol{\mu}$ ou por sua estimativa $\boldsymbol{y}_{\boldsymbol{i}}=\hat{\boldsymbol{\mu}}$, através de uma simplificação do ambiente estocástico. Sabe-se que os valores estimados $\boldsymbol{y}_{\boldsymbol{i}}=\hat{\boldsymbol{\mu}}$ não têm necessariamente que ocorrer, isto é, alguma diferença é esperada devida à variabilidade natural $\left(\boldsymbol{\varepsilon}_{i}\right)$ do ambiente florestal. Entretanto, o ponto estimado é tratado como um valor fixo, pois de fato ele é a melhor estimativa pontual que pode ser feita.

Estas preocupações relativas ao uso de modelos de decisão determinísticos em ambiente nãodeterminístico já foram consideradas em trabalhos como o de Pickens, Dress (1988). Trabalhos como o de Hof, Pickens (1991) e Hof, Kent, Pickens (1992) introduzem os conceitos de restrições probabilísticas, porém algoritmos da programação não-linear têm que ser usados para a solução dos mesmos. Weintraub, Barros (1996) enfatizam que é importante que gerentes florestais reconheçam os efeitos das incertezas quando decisões são tomadas no planejamento florestal.

Para quantificar o impacto que informações estocásticas produzem na resposta de modelos de planejamento usando programação linear, foram feitas simulações nas informações chaves, que foram consideradas variáveis aleatórias, tais como os coeficientes de produção, os custos de manejo, os preços e as demandas dos produtos, propiciando uma visão mais ampla dos possíveis valores que a receita líquida da empresa pode assumir. 
Esta abordagem da tomada de decisões amplia a informação do gerente, pois a resposta do sistema é dada através de uma distribuição de saídas da receita associada ao risco de obtê-la, em vez de um único valor determinístico para a função objetivo.

Foi feita uma comparação entre os resultados de um modelo básico, que é um modelo convencional que não considera as variações estocásticas das informações e os obtidos pelas novas técnicas que incorporam a estocasticidade na análise na tomada de decisões. Também foi avaliado o grau de risco que o responsável pelas decisões está sujeito em termos de resposta do sistema, através de um estudo de caso.

\section{O PROBLEMA FLORESTAL}

O modelo de planejamento florestal considerado baseou-se no modelo de Carnieri et al. (1991) e se refere a uma empresa florestal que atua no setor de papel. A empresa atende a demanda de madeira da fábrica de papel acoplada a ela, como também pode vender seus produtos florestais em caso de folga de produção. Na falta de madeira (polpa celulósica) para o processo de fabricação de papel, esta pode ser adquirida através de compra de terceiros ou por arrendamento.

O modelo contém as seguintes restrições:

\section{(1) Restrições de área}

Representando por $\boldsymbol{i}$ o estrato, $\boldsymbol{j}$ o regime de manejo, $\boldsymbol{N E}$ o número total de estratos, $\boldsymbol{N} \boldsymbol{R}_{\boldsymbol{i}} \mathrm{O}$ número máximo de regimes associados ao estrato $\boldsymbol{i}$ e $\boldsymbol{x}_{\boldsymbol{i} j}$ o número de hectares do estrato $\boldsymbol{i}$ a ser manejado segundo o regime $\boldsymbol{j}$, então

$$
x_{i 1}+x_{i 2}+\ldots+x_{i N R_{i}} \leq A R E A_{i}, \forall i=1, \ldots N E,
$$

onde $\boldsymbol{A R E} \boldsymbol{A}_{i}$ é a área plantada do estrato $\boldsymbol{i}$. O número possível de regimes por estrato depende da idade do mesmo e das atividades já desenvolvidas no estrato até então.

\section{(2) Restrições de compra de madeira para processo em todos os períodos}

Eventualmente, durante qualquer período de planejamento, representado por $\boldsymbol{k}$, a madeira a ser utilizada na produção de papel pode ser adquirida de outros, porém até um certo volume máximo que é definido pela empresa. Estas restrições são representadas por:

$$
C M P_{k} \leq V M C P_{k}, \forall k=1, \ldots P P,
$$

sendo que $\boldsymbol{C M} \boldsymbol{P}_{\boldsymbol{k}}$ representa a quantidade de madeira $\mathrm{em}^{3}$ a ser comprada para processo, no período $\boldsymbol{k} ; \boldsymbol{V} \boldsymbol{M C} \boldsymbol{P}_{\boldsymbol{k}}$, o volume máximo de madeira para processo $\mathrm{em}^{3}$ que pode ser adquirido no período $\boldsymbol{k}$ e $\boldsymbol{P P}$ o número máximo de períodos a serem considerados no planejamento.

(3) Restrições de demanda de cada produto florestal em todos os períodos

Os $\boldsymbol{N E}$ estratos considerados fornecem produtos que, em função da origem da área ( podada $\boldsymbol{p}$, não-podada $\boldsymbol{s} \boldsymbol{p}$ ) e de sua bitola comercial, são definidos como produtos laminados (mais nobres), produto para serraria, produto para processo de fabricação de papel e produto para energia (menos nobre). Ao todo foram considerados $\boldsymbol{N P}$ produtos.

Para descrever as restrições de demanda de cada produto $l$ é necessário considerar a demanda mínima $\boldsymbol{D}_{\boldsymbol{l} \boldsymbol{k}}$ exigida do produto $\boldsymbol{l}$ em cada período $\boldsymbol{k}$ de planejamento como também $\boldsymbol{D}_{\boldsymbol{L} \boldsymbol{k}}$ que é a demanda mínima do produto $\boldsymbol{L}$ que tem sobreposição de uso com o produto $\boldsymbol{l}$, no período $\boldsymbol{k}, \quad \boldsymbol{L}=\boldsymbol{l}_{1}, \ldots \boldsymbol{l}_{\boldsymbol{l}}$. Por sobreposição de uso entende-se a situação em que existindo demanda de um produto menos nobre $(\boldsymbol{l})$ e sobrando de um mais nobre $(\boldsymbol{L})$, este pode ser utilizado para suprir a demanda devida. Assim sendo, a forma das inequações de demanda para cada produto $l$ pode ser descrita por: 


$$
\sum_{i=1}^{N E} \sum_{j=1}^{N R_{i}} a_{i j}^{l k} \cdot x_{i j}-\sum_{L=l_{1}}^{L=l_{l}} V_{L k} \geq \sum_{L=l_{1}}^{L=l_{l}} D_{L k}, \forall k=1, \ldots P P
$$

onde a variação do índice $\boldsymbol{L}$, no somatório das vendas do produto $\boldsymbol{L}$ no período $\boldsymbol{k}\left(\boldsymbol{V}_{\boldsymbol{L} \boldsymbol{k}}\right)$ e no somatório das demandas do produto $\boldsymbol{L}$ no período $\boldsymbol{k}\left(\boldsymbol{D}_{\boldsymbol{L} \boldsymbol{k}}\right)$, depende do produto $\boldsymbol{l}$ que está sendo considerado na restrição. O coeficiente $\boldsymbol{a}_{\boldsymbol{i j}}^{\boldsymbol{l k}}$ representa a produção em $\mathrm{m}^{3} /$ ha. de madeira obtida no estrato $\boldsymbol{i}$, com regime de manejo $\boldsymbol{j}$, do produto $\boldsymbol{l}$, no período $\boldsymbol{k}$.

As restrições de demanda dos produtos considerados, em todos os períodos de planejamento, foram desenvolvidas de forma a atender a duas condições, conforme em Volpi (1997): sobreposição de uso e foi evitado que um mesmo volume seja utilizado mais de uma vez pois existe interseção de utilização entre os produtos.

(4) Restrições de volume máximo desbastado

Em geral são necessárias restrições que evitem que haja um volume de corte muito acentuado nos primeiros anos de planejamento, já que o valor líquido presente usado na função objetivo prioriza as receitas obtidas nestes primeiros anos. Este conjunto de restrições também é utilizado quando existe restrição técnica em termos de capacidade de produção da fábrica em utilizar a produção de madeira explorada; quando as empresas possuem muitas áreas com árvores de maior porte disponíveis para corte ou mesmo quando se quer controlar o total disponível de madeira no mercado.

Limita-se a produção global nos $\boldsymbol{P}$ primeiros períodos de planejamento através das inequações:

$$
\sum_{i=1}^{N E} \sum_{j=1}^{N R_{i}} a_{i j}^{10 k} \cdot x_{i j} \leq V M A X_{k}, \forall k=1, \ldots P
$$

onde $\boldsymbol{V} \boldsymbol{M} \boldsymbol{A} \boldsymbol{X}_{\boldsymbol{k}}$ representa o volume máximo em $\mathrm{m}^{3}$ permitido para corte no período $\boldsymbol{k}$ e $\boldsymbol{a}_{\boldsymbol{i j}}^{\mathbf{1 0 k}}$ a produção máxima de madeira $\mathrm{em}^{3} /$ ha. no período $\boldsymbol{k}$, do estrato $\boldsymbol{i}$, segundo o regime $\boldsymbol{j}$.

\section{Função objetivo}

O objetivo considerado no problema foi o de maximizar o valor líquido presente $(\boldsymbol{V L P})$ da renda total ao longo de todo o horizonte de planejamento.

A receita líquida total depende da receita devida aos contratos já efetuados, que são as demandas obrigatórias $(\boldsymbol{R D} \boldsymbol{O})$, das vendas dos produtos a terceiros $\left(\boldsymbol{R}_{\boldsymbol{V}}\right)$, dos custos de manejo $\left(\boldsymbol{C}_{\boldsymbol{M}}\right)$, como também da compra de madeira para processo $\left(\boldsymbol{C}_{\boldsymbol{C M P}}\right)$, permitida em caso de necessidade. Um valor terminal $(\boldsymbol{V T})$ associado a cada estrato $\boldsymbol{i}$ e regime de manejo $\boldsymbol{j}$ foi acrescentado ao $\boldsymbol{V L P}$, supondo que após o término do horizonte de planejamento cada estrato será manejado em perpetuidade, segundo seu regime economicamente ótimo $(\boldsymbol{R E} \boldsymbol{O})$. O $\boldsymbol{R E} \boldsymbol{O}$ é aquele regime entre os disponíveis, que produz a melhor renda líquida numa rotação. Uma rotação florestal representa o período entre plantio e corte final. $\mathrm{O}$ valor terminal $\boldsymbol{V} \boldsymbol{T}$ considera a possibilidade futura do uso da madeira e o $\boldsymbol{V L P}$ calculado desta forma é chamado de valor líquido presente geral.

Portanto, tem-se para função objetivo:

$$
\begin{aligned}
\max z=R D O & +\sum_{k=1}^{P P} \sum_{l=1}^{N P} \frac{P V_{l k}}{(1+j u r o)^{k}} \cdot V_{l k}-\sum_{i=1}^{N E} \sum_{j=1}^{N R_{i}}\left(c_{i j}-V T_{i j}\right) \cdot x_{i j} \\
& -\sum_{k=1}^{P P} \frac{P C_{k}}{(1+j u r o)^{k}} \cdot C M P_{k}-\sum_{k=1}^{P P} \sum_{l=1}^{N P} M . V A_{l k},
\end{aligned}
$$


onde $\boldsymbol{P} \boldsymbol{V}_{\boldsymbol{l} \boldsymbol{k}}$ é o preço $/ \mathrm{m}^{3}$ de venda do produto $\boldsymbol{l}$ no período $\boldsymbol{k}, \boldsymbol{P} \boldsymbol{C}_{\boldsymbol{k}}$ o preço $/ \mathrm{m}^{3}$ de compra da madeira para processo no período $\boldsymbol{k}$, juro a taxa de juros utilizada no planejamento, $\boldsymbol{M}$ um valor grande utilizado no método Big-M, $\boldsymbol{V} \boldsymbol{A}_{\boldsymbol{l} \boldsymbol{k}}$ variáveis artificiais associadas as restrições de demandas do produto $\boldsymbol{l}$, no período $\boldsymbol{k}$ e os demais coeficientes e variáveis como definido anteriormente.

O modelo de planejamento florestal descrito é um problema de programação linear da forma:

$\max c \cdot x$

$$
A \cdot \boldsymbol{x}=b, x \geq 0,
$$

onde as variáveis de decisão são o número de hectares do estrato $\boldsymbol{i}$ manejado segundo $\boldsymbol{j}\left(\boldsymbol{x}_{\boldsymbol{i} j}\right)$, a compra de madeira para processo em $\boldsymbol{k}\left(\boldsymbol{C M P}_{\boldsymbol{k}}\right)$ e a venda do produto $\boldsymbol{l}$ em $\boldsymbol{k}\left(\boldsymbol{V}_{\boldsymbol{l}}\right)$. Para implementá-lo são necessárias algumas informações obtidas de ambientes estocásticos ou futuros, tais como:

1. demandas dos produtos $\boldsymbol{D}_{\boldsymbol{l} \boldsymbol{k}}$ considerados em todo o horizonte de planejamento;

2. coeficientes de produção $\boldsymbol{a}_{\boldsymbol{i} \boldsymbol{j}}^{\boldsymbol{l}}$;

3. custos de manejo $c_{i j}$;

4. preços dos produtos comercializados $\boldsymbol{P} \boldsymbol{V}_{\boldsymbol{l}}$ em todo o horizonte de planejamento.

Coeficientes de produção estocásticos geram matriz tecnológica e função objetivo estocásticas. Preços e custos estocásticos, em geral, só interferem na função objetivo. Demandas estocásticas interferem no vetor dos recursos $\boldsymbol{b}$.

A matriz $\boldsymbol{A}$ é formada pelos elementos $\mathbf{1}, \mathbf{- 1}, \mathbf{0}$ e $\boldsymbol{a}_{\boldsymbol{i}}^{\boldsymbol{l} \boldsymbol{k}}$, sendo que $\boldsymbol{a}_{\boldsymbol{i} j}^{\boldsymbol{l k}}$ é obtida por amostragem através de um valor estimado $\hat{\boldsymbol{a}}_{\boldsymbol{i} \boldsymbol{j}}^{\boldsymbol{l} \boldsymbol{k}}$.

$\mathrm{O}$ vetor $\boldsymbol{c}$ depende de valores de mercado $\boldsymbol{P} \boldsymbol{V}_{\boldsymbol{l}}, \boldsymbol{P} \boldsymbol{C}_{\boldsymbol{k}}$ e de $\boldsymbol{c}_{i j}$, sendo que apenas sobre os custos a empresa tem um certo controle.

$\mathrm{O}$ vetor $\boldsymbol{b}$ depende de informações das áreas plantadas, de contratos a serem atendidos de cada produto florestal, de informações econômicas e operacionais que indicam o quanto pode ser comprado de madeira para processo e o máximo que pode ser cortado em cada ano. Valores estimados também são considerados neste caso. O usual então é resolver o problema $(\boldsymbol{P L})$, considerando os valores esperados destas informações, tal como: $F O=\max _{x}\{E(c) . x / E(A) . x=E(b), x \geq 0\}$.

Uma análise de sensibilidade em $\boldsymbol{E}(\boldsymbol{c})$ e $\boldsymbol{E}(\boldsymbol{b})$ pode ser feita de modo a analisar a variabilidade de $\boldsymbol{F} \boldsymbol{O}$, porém na matriz $\boldsymbol{E}(\boldsymbol{A})$ a situação é bem mais complicada.

Para se obter mais informações da possível variabilidade de $\boldsymbol{F} \boldsymbol{O}$ perante à variabilidade das informações, usou-se:

a) simular a variabilidade estocástica das informações;

b) resolver o $(\boldsymbol{P L})$ perturbado através do SIMPLEX REVISADO e do GUB (Generalised Upper Bounding).

O GUB, conforme Dantzig (1967), foi utilizado devido a forma particular da matriz tecnológica $\boldsymbol{A}$ apresentada a seguir, onde $\boldsymbol{M}_{\boldsymbol{0}}$ é uma matriz formada por $\mathbf{1 , - 1}$ e zeros e $\boldsymbol{M}_{\boldsymbol{i}}, \boldsymbol{i}=\mathbf{1}, \ldots \boldsymbol{N} \boldsymbol{E}$ uma matriz formada por coeficientes de produção $\boldsymbol{a}_{\boldsymbol{i} j}^{\boldsymbol{l} \boldsymbol{k}}$ : 


$$
A=\left[\begin{array}{cccccccccc}
1 & \ldots & 1 & & & & & - & \\
& & & \ldots & & & & - & 0 \\
& & & & 1 & \ldots & 1 & - & \\
- & - & - & - & - & - & - & - & - \\
& M_{1} & & \ldots & & M_{N E} & & - & M_{0}
\end{array}\right]
$$

O uso do GUB evita que se trabalhe com a matriz inteira $A$, mas com apenas parte dela, que após uma transformação de variáveis é chamada de matriz de trabalho.

O processo de simulação, desenvolvido neste trabalho:

a) Estabeleceu distribuições de probabilidades para os valores das informações a serem simuladas em função do comportamento passado destes dados.

b) Definiu o risco, através do coeficiente de variação $\boldsymbol{C V}$ da amostra obtida.

Através de análise de situações reais ocorreu a necessidade de se avaliar 3 casos:

1. Caso onde os valores medidos variam em torno da média esperada, com valores concentrando-se em torno desta média com maior probabilidade do que longe dela. Considerando-se várias situações reais possíveis, cada variável aleatória foi perturbada com ruído normal de acordo com diversos coeficientes de variação. Este é considerado o caso mais comum.

2. Caso onde os dados variam dentro de um intervalo com a mesma probabilidade. Foi usado o modelo de distribuição uniforme, com diversas amplitudes, para simular várias situações possíveis. É considerado o caso mais crítico.

3. Caso onde os dados são obtidos de uma forma tendenciosa: ou para mais ou para menos do valor real. Nesta situação os dados foram alterados sistematicamente, sendo multiplicados por um valor constante.

Foi desenvolvido um simulador específico para trabalhar com tais situações, o SIMULA, com a possibilidade de se escolher o tipo e o nível da perturbação.

Foram feitas simulações, perturbando-se isoladamente o vetor $\boldsymbol{c}$, o $\boldsymbol{b}$ e a matriz $\boldsymbol{A}$ (e consequentemente o $\boldsymbol{c}$, que depende das informações de $\boldsymbol{A}$ ). Em cada situação foi resolvido o problema de $\boldsymbol{P L}$ e observada a variável aleatória $\boldsymbol{F} \boldsymbol{O}_{p e r t}$, definida por:

$$
\begin{aligned}
& F O_{\text {pert }}=\max _{x}\left\{c_{p e r t} \cdot x / A . x=b, x \geq 0\right\}, \quad F O_{\text {pert }}=\max _{x}\left\{c \cdot x / A . x=b_{p e r t}, x \geq 0\right\}, \\
& F O_{\text {pert }}=\max _{x}\left\{c_{\text {pert }} . x / A_{\text {pert }} . x=b, x \geq 0\right\} \text {, respectivamente }
\end{aligned}
$$

Foram feitas comparações com o valor de

$$
F O_{\text {básico }}=F O=\max _{x}\{E(c) . x / E(A) . x=E(b), x \geq 0\},
$$

que representa o retorno financeiro quando a informação média está disponível.

Sabe-se que $\boldsymbol{E}\left(\boldsymbol{F} \boldsymbol{O}_{\text {pert }}\right)$ não é necessariamente igual a $\boldsymbol{F} \boldsymbol{O}_{\text {básico }}$, conforme Falácia das Médias e Teorema da Equivalência Linear, apresentados em Wagner (1986). Além do mais, a equação y $=\boldsymbol{E}\left(\boldsymbol{F} \boldsymbol{O}_{\text {pert }}\right)$ sujeito as restrições devidamente perturbadas admite uma solução que não está associada a nenhum valor tal como o valor esperado das soluções e do valor esperado dos ótimos de cada $\boldsymbol{P L}$ perturbado. 
Quando $\boldsymbol{A}$ e $\boldsymbol{c}$ são estocásticos, a situação é mais complexa.

A prática corrente de usar as estimativas dos valores esperados para as informações que são variáveis aleatórias, pode gerar aproximações para $\boldsymbol{E}\left(\boldsymbol{F} \boldsymbol{O}_{\text {pert }}\right)$, porém pode levar a soluções que possuem baixa probabilidade de serem factíveis.

Considerando-se que as estimativas dos valores esperados sejam a informação perfeita e usando-se os valores perturbados segundo alguma distribuição em torno desta média, pode-se analisar como os valores de $\boldsymbol{x}$ e de $\boldsymbol{F} \boldsymbol{O}$ alterariam e qual seria o risco, em função de onde foi feita a perturbação e do grau da perturbação.

O programa SIMULA pode ser utilizado quando se quiser perturbar qualquer variável aleatória que consta dos arquivos de área, demanda, preço, custo de manejo, volume máximo de compra de madeira para processo, volume de controle de corte global ou coeficiente de produção.

A idéia básica é considerar o parâmetro que se quer tornar aleatório e perturbá-lo segundo um ruído com distribuição escolhida ou através de uma perturbação sistemática e depois colocar os dados alterados pela perturbação em outro arquivo, para daí usar o SIMPLEX com os dados perturbados.

O sistema SIMULA é composto dos seguintes programas:

$\begin{array}{lll}\text { SIMULA } & \Rightarrow & \begin{array}{l}\text { Programa principal. } \\ \text { CARQS( ) }\end{array} \\ \text { CRIAESPS( ) } & \Rightarrow \quad \begin{array}{l}\text { Subrotina para calcular os custos de manejo em } \\ \text { função da produção global. }\end{array} \\ \text { RESOLVES( ) } & \Rightarrow \quad \begin{array}{l}\text { Subrotina que gera automaticamente os arquivos } \\ \text { de coeficientes de produção. }\end{array} \\ & & \text { e o GUB } .\end{array}$

\section{RESULTADOS}

\subsection{UM ESTUDO DE CASO}

O modelo de planejamento desenvolvido na seção anterior foi executado, utilizando-se dados de uma empresa florestal em aproximadamente 13.000 ha. de reflorestamento de Pinus taeda.

Foram considerados 80 estratos, distribuídos em 5 regiões de diferentes distâncias médias ao centro de operações, onde está localizada a administração e a fábrica de papel. Considerou-se no máximo 12 regimes por estrato. O modelo contempla 10 diferentes produtos madeireiros: madeira para laminado tipo 1,2 e 3 , podados e não-podados; madeira para serraria, podada e não-podada, madeira para fabricação de papel e madeira para ser usada como energia.

O planejamento foi desenvolvido para um horizonte de 30 anos com períodos de um ano. Os primeiros 5 anos de planejamento possuem controle de volume total de corte. A matriz tecnológica $\boldsymbol{A}$ para este caso tem dimensão 415 x 1925.

Os dados utilizados na formação do modelo estão na forma de arquivos, os quais são: arquivo das áreas disponíveis dos 80 estratos; arquivo de demandas de cada produto em cada período; arquivo de preços de cada produto em todos os períodos de planejamento; arquivo de volume máximo de compra de madeira para processo em função de um levantamento da disponibilidade de madeira no mercado; arquivo de controle de volume de corte, informando o máximo que pode ser cortado nos primeiros $\boldsymbol{P}$ anos de planejamento, em $\mathrm{m}^{3}$. Outros arquivos 
também são necessários: o arquivo de custos de manejo, de valores terminais e de coeficientes de produção.

\section{$\underline{\text { Arquivo de custos de manejo }}$}

Para executar o modelo descrito se faz necessário conhecer também cada custo $c_{i j}$ das atividades do manejo $\boldsymbol{j}$, do estrato $\boldsymbol{i}$, quando implementado em uma unidade de área. Foram considerados 3 grupos distintos de regimes de manejo: regimes clearwood, cujo objetivo é manejar através da retirada de galhos para melhorar a qualidade da madeira; regimes utility, cujo objetivo é manejar para obter produtos de serraria e laminados, além de madeira para processo; e regimes pulpwood cuja principal meta é obter madeira para atender o processo de fabricação de papel. Além desses regimes foi considerado mais um, disponível a todos os estratos, chamado de regime 'abandono'. Este regime, se escolhido, significa que a área será deixada como estava no início do planejamento e considerada desnecessária para a empresa, no sentido que todas as demandas obrigatórias são atendidas sem precisar explorar a área considerada e que se manejada trará prejuízo. $\mathrm{O}$ cálculo dos custos foi feito em função dos custos operacionais, que se referem aos dispêndios diretos na produção. Nas atividades terceirizadas os custos são aqueles pagos aos empreiteiros.

\section{Arquivo de valores terminais}

O valor terminal $\boldsymbol{V} \boldsymbol{T}_{i j}$ relativo ao estrato $\boldsymbol{i}$ e manejo $\boldsymbol{j}$ representa um fluxo de caixa obtido pela aplicação do regime economicamente ótimo $(\boldsymbol{R E} \boldsymbol{O})$ no estrato $\boldsymbol{i}$, após o final do período de planejamento, considerando as hipóteses apresentadas por Clutter et al.(1983) as quais são:

- Se a idade do estrato $\boldsymbol{i}_{F}$ no final do período de planejamento é maior ou igual a idade do corte raso do $\boldsymbol{R E} \boldsymbol{O}$, o estrato será cortado ao final deste período.

- Se a idade $\boldsymbol{i}_{F}$ do estrato no final do período de planejamento é menor que a do corte raso do $\boldsymbol{R E} \boldsymbol{O}$, então maneja-se o estrato segundo o $\boldsymbol{R E \boldsymbol { O }}$ até o corte raso, fornecendo um fluxo financeiro, cujo valor presente no final do planejamento será chamado de Valor do Estoque em Pé $(\boldsymbol{V E \boldsymbol { E }})$.

- Após o corte do estrato $\boldsymbol{i}$, supõe-se que o mesmo será manejado pelo seu $\boldsymbol{R E} \boldsymbol{O}$ em perpetuidade, cujo fluxo de caixa será chamado de Valor Esperado da Terra (VET).

\section{Arquivo de coeficientes de produção}

Muitas restrições do modelo florestal dependem de dados relativos aos coeficientes de produção dos estratos considerados. As produções de cada um dos 10 produtos considerados, dependem, por sua vez, das informações desses estratos, como do seu índice de sítio $s$, sua densidade inicial de plantio $\boldsymbol{d}$, da região $\boldsymbol{r}$ onde está localizado o estrato, da característica de ser podado $(\boldsymbol{P})$ ou não-podado $(\boldsymbol{N})$ e do regime de manejo $\mathbf{r e g}$.

Foi utilizado o simulador de crescimento e produção, SISPINUS, conforme Oliveira et al. (1991), para gerar os coeficientes de produção. O programa CRIAESPS coloca estas informações na forma a ser lida no RESOLVES. 


\subsection{RESULTADOS DO ESTUDO DE CASO}

Os dados que alimentam o modelo florestal considerado foram perturbados por diferentes distribuições de probabilidades e vários graus de variabilidade. Três situações foram consideradas e avaliado sua influência na função objetivo:

1. Distribuição normal com coeficiente de variação $\boldsymbol{C} \boldsymbol{V}=10,20$ e $30 \%$, representando a situação mais comum;

2. Situação média, com valores esperados dos dados;

3. Distribuição uniforme, com semi-amplitudes de $1 / 3,1 / 2$ e $2 / 3$ em relação ao valor médio, representando uma situação mais crítica.

\subsubsection{Simulações normais:}

Cada coeficiente de produção $\boldsymbol{a}_{\boldsymbol{i} j}^{\boldsymbol{l} \boldsymbol{k}}$ foi perturbado por um ruído com uma distribuição normal, da forma $a_{p e r t}=a_{i j}^{l k}+n \cdot \sigma$, para diferentes coeficientes de variação $\boldsymbol{C} \boldsymbol{V}$ e $\boldsymbol{n} \sim \boldsymbol{N}(\mathbf{0 , 1})$, tal que o valor perturbado $\boldsymbol{a}_{p e r t} \sim N\left(\boldsymbol{a}_{\boldsymbol{i j}}^{\boldsymbol{l k}}, \boldsymbol{\sigma}^{2}\right)$. O desvio padrão $\boldsymbol{\sigma}$ pode ser estimado por $\boldsymbol{s}=\boldsymbol{a}_{\boldsymbol{i j}}^{\boldsymbol{l k}} . \boldsymbol{C} \boldsymbol{V}$ e o valor perturbado é descrito por $a_{p e r t}=a_{i j}^{l k}+n \cdot s=a_{i j}^{l k}+n \cdot a_{i j}^{l k} \cdot C V=a_{i j}^{l k} \cdot(1+n \cdot C V)$. Para cada nível de variação nos coeficientes de produção obteve-se uma amostra de 100 valores da função objetivo $\boldsymbol{F O}_{\text {pert }}$, cujas estatísticas estão na Tabela 01 . O valor $\overline{\boldsymbol{F O}}_{\text {pert }}$ representa a média dos 100 valores da amostra e o valor de $\overline{\boldsymbol{F O}}_{\text {pert }}$ para $\boldsymbol{C} \boldsymbol{V}=0 \%$ representa o ótimo quando valores esperados são utilizados no modelo $\left(\overline{\boldsymbol{F O}}_{\left.\text {pert }_{(} \boldsymbol{C V}=0 \%\right)}=\boldsymbol{F O}_{\text {básico }}\right)$.

TABELA 01: ESTATÍSTICAS DA AMOSTRA DE FUNÇÕES OBJETIVOS COM PERTURBAÇÕES NORMAIS NOS DADOS DE PRODUÇÃO

\begin{tabular}{|c|c|c|c|c|}
\hline $\boldsymbol{C} \boldsymbol{V}$ em $a_{i j}^{l k}$ & $0 \%$ & $10 \%$ & $20 \%$ & $30 \%$ \\
\hline$\overline{F O}_{\text {pert }}(\mathrm{US} \$)$ & $49.667 .472,20$ & $49.682 .040,90$ & $50.624 .533,60$ & $49.892 .906,80$ \\
\hline Desvio padrão(US\$) & 0,00 & $2.692 .947,12$ & $4.354 .311,58$ & $6.551 .788,26$ \\
\hline$C V$ da amostra de $F O_{p e r t}(\%)$ & 0,00 & 5,42 & 8,60 & 13,13 \\
\hline Valor mínimo (US\$) & 49.667.472,20 & $42.872 .266,50$ & $41.816 .182,30$ & $34.614 .367,30$ \\
\hline Valor máximo (US\$) & 49.667.472,20 & $56.051 .791,80$ & $59.680 .802,30$ & $65.280 .654,50$ \\
\hline Amplitude (US\$) & 0,00 & $13.179 .525,30$ & $17.864 .620,00$ & $30.666 .287,20$ \\
\hline $\begin{array}{l}\text { Semi-amplitude do intervalo } \\
\text { confianca média (US\$) }\end{array}$ & 0,00 & $527.807,16$ & $853.428,12$ & $1.284 .125,00$ \\
\hline
\end{tabular}

Através de um teste de hipóteses sobre o valor médio das amostras obtidas para cada nível de variação, verificou-se que, em média, os valores da receita encontrada não diferem significativamente do valor encontrado quando se usam as estimativas dos valores esperados de produção. Este é um bom resultado, no sentido de que tem-se $\boldsymbol{E}\left(\boldsymbol{F} \boldsymbol{O}_{\text {pert }}\right) \cong \boldsymbol{F} \boldsymbol{O}_{\text {básico }}$.

A dispersão dos valores de $\boldsymbol{F} \boldsymbol{O}_{\text {pert }}$ tende a aumentar de uma forma quase linear, com o aumento dos coeficientes de variação dos dados de produção. O risco pode ser avaliado através dos valores de $\boldsymbol{C} \boldsymbol{V}$ das amostras de $\boldsymbol{F} \boldsymbol{O}_{\text {pert }}$ apresentados na Tabela 01 . 
Através do teste de Filliben, conforme Filliben (1975), verificou-se que os valores de $\boldsymbol{F O}_{\text {pert }}$ obtidos nas simulações pertencem a uma distribuição normal. Pelo fato das saídas serem normais é garantido o uso de todas as propriedades da distribuição normal, podendo-se fazer inferências paramétricas sobre esta variável. Conclui-se que o resultado das simulações fornece uma base mais adequada para tomada de decisões, sendo que o gerente responsável pelas decisões tem uma infinidade de posições risco $\mathbf{x}$ retorno financeiro para considerar, em vez de um único ponto estimado $\left(\boldsymbol{F} \boldsymbol{O}_{\text {básico }}\right)$. Os valores de $\boldsymbol{F} \boldsymbol{O}_{\text {pert }}$ acima do valor $\boldsymbol{F} \boldsymbol{O}_{\text {básico }}$ oferecem vantagem para a empresa, porém valores abaixo dele representam perda no valor final estimado no planejamento e devem ser examinadas com cuidado.

No planejamento de uma empresa florestal, as informações obtidas por simulação com base em dados amostrais reais é extremamente importante, já que seria impossível repetir o planejamento inúmeras vezes, ou em inúmeras empresas ao mesmo tempo e nas mesmas condições. A simulação faz este papel, contribuindo com os valores possíveis da receita, o seu valor médio e desvio padrão estimados. Então, dependendo do grau de informação que o gerente possui em relação à variabilidade dos coeficientes de produção, em relação à média considerada $\boldsymbol{a}_{\boldsymbol{i} \boldsymbol{j}}^{\boldsymbol{l k}}$, ele pode ter uma visão concreta do risco que está correndo em termos de receita líquida no empreendimento florestal, através da distribuição $N\left(\overline{F O}_{\text {pert }}, C V \cdot \overline{F O}_{\text {pert }}\right)$.

\subsubsection{Simulações uniformes:}

No caso das simulações uniformes cada coeficiente de produção foi perturbado segundo a equação $\boldsymbol{a}_{i j \text { pert }}^{l k}=\boldsymbol{a}_{i j}^{l k}+\boldsymbol{a}_{i j}^{l k} \cdot(\mathbf{2} \boldsymbol{R}-\mathbf{1}) \cdot \boldsymbol{t}$, sendo $\boldsymbol{t} \boldsymbol{\varepsilon}[\mathbf{0 , 1}]$ e $\boldsymbol{a}_{i j}^{\boldsymbol{l k}}$ uma estimativa do valor

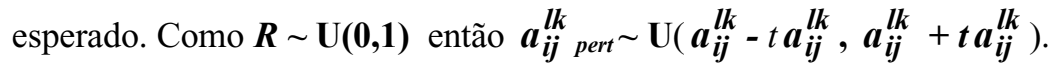

Para cada amplitude $\left(\mathbf{2} \boldsymbol{t} \boldsymbol{a}_{\boldsymbol{i} \boldsymbol{j}}^{\boldsymbol{k}}\right)$ considerada na perturbação dos coeficientes de produção $\boldsymbol{a}_{\boldsymbol{i} \boldsymbol{j}}^{\boldsymbol{k}}$ através da distribuição uniforme, observaram-se numa amostra de 100 simulações as estatísticas apresentadas na Tabela 02.

TABELA 02: ESTATÍSTICAS DA AMOSTRA DE FUNÇÕES OBJETIVOS COM PERTURBAÇÕES UNIFORMES EM DADOS DE PRODUÇÃO

\begin{tabular}{|c|c|c|c|c|}
\hline $\begin{array}{l}\text { Amplitude } \\
\left(\mathbf{2} \cdot \boldsymbol{t} \cdot \mathbf{a}_{\mathrm{ij}}\right)\end{array}$ & $\begin{array}{l}\text { Amplitude } \\
0\end{array}$ & $\begin{array}{l}\text { Amplitude } \\
2.1 / 3 \cdot a_{i j}\end{array}$ & $\begin{array}{l}\text { Amplitude } \\
2.1 / 2 \cdot a_{i j}\end{array}$ & $\begin{array}{l}\text { Amplitude } \\
2.2 / 3 \cdot a_{i j}\end{array}$ \\
\hline$\overline{F O}_{\text {pert }}(\mathrm{US} \$)$ & $49.667 .472,20$ & $49.691 .234,50$ & $48.882 .285,60$ & $49.547 .575,00$ \\
\hline Desvio padrão(US\$) & 0,00 & $3.417 .224,62$ & $4.771 .649,93$ & $6.767 .870,26$ \\
\hline$C V$ da amostra de $F O_{\text {pert }}(\%)$ & 0,00 & 6,90 & 9,76 & 13,70 \\
\hline Valor mínimo(US\$) & $49.667 .472,20$ & $42.054 .392,20$ & $37.607 .545,40$ & $33.847 .372,80$ \\
\hline Valor máximo(US\$) & $49.667 .472,20$ & $56.959 .488,50$ & $59.102 .435,10$ & $67.085 .577,50$ \\
\hline Amplitude (US\$) & 0,00 & $14.905 .096,30$ & $21.494 .889,70$ & $33.238 .204,70$ \\
\hline $\begin{array}{l}\text { Semi-amplitude do intervalo } \\
\text { confiança média (US\$) }\end{array}$ & 0,00 & $669.762,73$ & $935.224,82$ & $1.326 .476,23$ \\
\hline
\end{tabular}

Fazendo um teste de hipóteses sobre as médias calculadas, encontrou-se que não existe diferença significativa entre os valores obtidos para as médias das perturbações e o valor $\boldsymbol{F} \boldsymbol{O}_{\text {básico }}$ do modelo básico. Neste caso também a dispersão dos valores de $\boldsymbol{F O}_{\text {pert }}$ tende a aumentar de uma forma quase linear, com o aumento das amplitudes da distribuição uniforme 
dos dados de produção, conforme se observa nos valores de $\boldsymbol{C} \boldsymbol{V}$ da amostra de $\boldsymbol{F}_{\text {pert }}$ na Tabela 02 .

As amostras em cada situação foram testadas quanto ao nível de normalidade, através do teste de Filliben e foram encontradas normais. Conclui-se também que independente de como os dados de produção tenham sido perturbados, seja através de uma distribuição normal ou uniforme, a distribuição de saída das funções objetivos, para o modelo que está sendo analisado, é normal.

Sendo a perturbação uniforme mais crítica em termos de variabilidade do que a simulação normal, observa-se que os coeficientes de variação da função objetivo são maiores que os obtidos na situação anterior. Os limites de variação possíveis de $\boldsymbol{F} \boldsymbol{O}_{\text {pert }}$ também aumentaram como era previsível, conforme pode ser observado nas amplitudes das distribuições, na Tabela 02 .

\subsubsection{Cenários sistemáticos:}

Os cenários testados foram o de reduzir e aumentar sistematicamente todos os coeficientes de produção da matriz tecnológica $\boldsymbol{A}$, da forma $\boldsymbol{a}_{\boldsymbol{i j} \text { pert }}^{\boldsymbol{k}}=\boldsymbol{A L F A} \cdot \boldsymbol{a}_{\boldsymbol{i j}}^{\boldsymbol{k} \boldsymbol{k}}$, onde $\boldsymbol{A L F A}$ é um valor constante. Esta situação ocorre com uma certa freqüência devido a erros de medições em instrumentos descalibrados ou mesmo falha sistemática humana na medição. Os valores da função objetivo, para alguns valores de $\boldsymbol{A L F \boldsymbol { A }}$ estão representados na Tabela 03. Observa-se

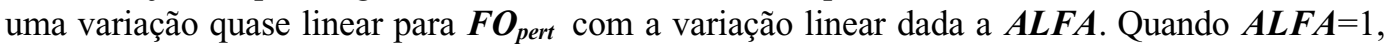
$\boldsymbol{F} \boldsymbol{O}_{\text {pert }}=\boldsymbol{F} \boldsymbol{O}_{\text {básico. }}$

\subsubsection{Perturbações nos outros arquivos}

Toda a análise feita anteriormente para os coeficientes de produção também foi realizada para as informações de custos de manejo, preços dos produtos e demandas dos produtos. Os valores das funções objetivos médias estão representadas na Figura 01. Na Tabela 04 observam-se os coeficientes de variação encontrados para cada situação simulada. Observa-se uma nítida diferença em cada situação, informando por exemplo, que os preços devem ser obtidos de forma mais cuidadosa que as demandas.

TABELA 03: VALORES DE $\boldsymbol{F}_{\boldsymbol{O}_{\text {pert }}}$ PARA VARIAÇÕES SISTEMÁTICAS EM DADOS DE PRODUÇÃO

\begin{tabular}{|l|l|l|}
\hline $\boldsymbol{A L F A}$ & Função Objetivo (US\$) & Diferença entre valores de $\boldsymbol{F} \boldsymbol{O}_{\text {pert }}$ (US\$) \\
\hline 0,5 & $22.670 .956,00$ & \\
0,6 & $28.091 .532,00$ & $5.420 .576,00$ \\
0,7 & $33.510 .753,00$ & $5.419 .221,00$ \\
0,8 & $38.910 .376,00$ & $5.399 .623,00$ \\
0,9 & $44.290 .396,00$ & $5.380 .020,00$ \\
1,0 & $49.667 .472,00$ & $5.377 .076,00$ \\
1,1 & $55.035 .007,00$ & $5.376 .535,00$ \\
1,2 & $60.366 .527,00$ & $5.331 .520,00$ \\
1,3 & $65.712 .269,00$ & $5.345 .742,00$ \\
1,4 & $71.015 .392,00$ & $5.303 .123,00$ \\
1,5 & $76.380 .805,00$ & $5.365 .413,00$ \\
\hline
\end{tabular}


FIGURA 01: VALORES DAS FUNÇÕES OBJETIVOS MÉDIAS DE CADA AMOSTRA (SIMULAÇÃO NORMAL)

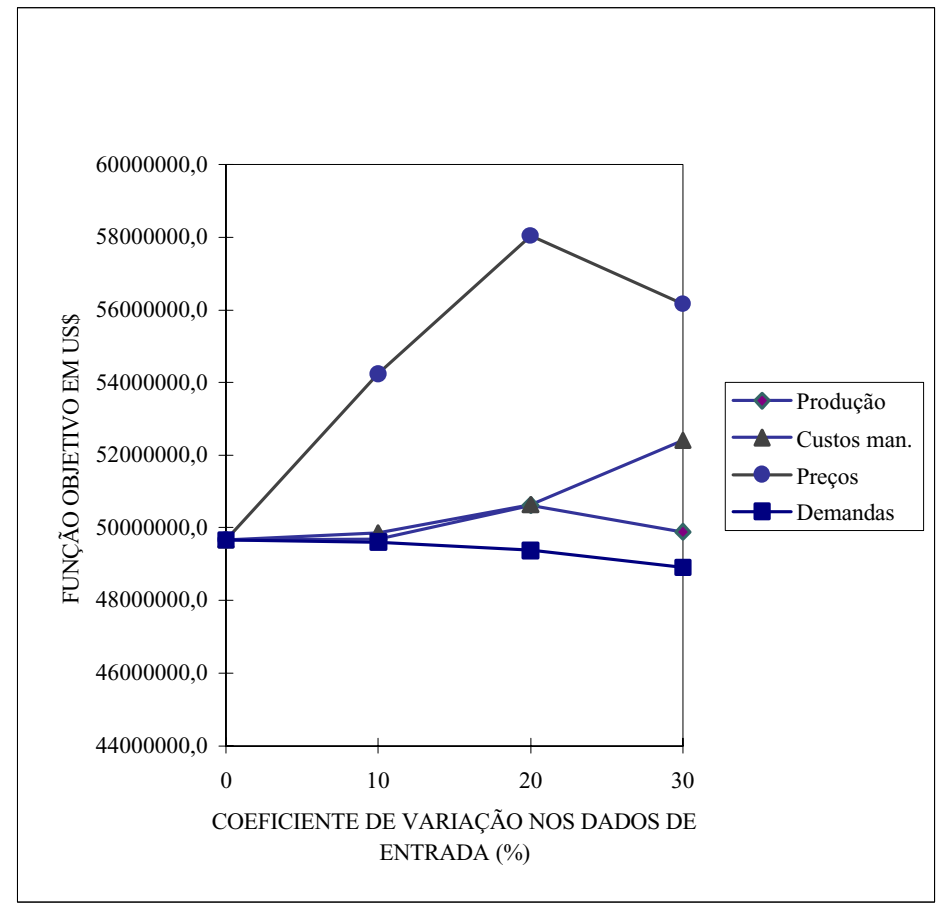

TABELA 04: COEFICIENTES DE VARIAÇÃO DAS AMOSTRAS DE $\boldsymbol{F}_{\boldsymbol{p} \text { ert }}$

\begin{tabular}{|l|l|c|c|l|l|}
\hline & Objeto & $\boldsymbol{a}_{\mathrm{ij}}(\%)$ & $\boldsymbol{c}_{\mathrm{ij}}(\%)$ & $\boldsymbol{P} \boldsymbol{V}_{\mathbf{l k}}(\%)$ & $\boldsymbol{D}_{\mathbf{l k}}(\%)$ \\
\hline & $C V=10 \%$ & 5,4 & 3,6 & 13,4 & 0,2 \\
SIMULAÇÃO & $C V=20 \%$ & 8,6 & 7,2 & 25,9 & 0,6 \\
NORMAL & $C V=30 \%$ & 13,1 & 10,6 & 29,4 & 1,9 \\
\hline & $\mathrm{t}=1 / 3$ & 6,9 & & & \\
SIMULAÇÃO & $\mathrm{t}=1 / 2$ & 9,8 & & & \\
UNIFORME & $\mathrm{t}=2 / 3$ & 13,7 & & & \\
\hline
\end{tabular}

Verificou-se que apenas os valores médios da função objetivo para perturbações em coeficientes de produção e custos com $C V=10 \%$, são estatisticamente iguais ao valor obtido no modelo básico ( $\boldsymbol{C} \boldsymbol{V}=0 \%$ ). O risco pode ser medido pelo $\boldsymbol{C} \boldsymbol{V}$ da amostra de valores de $\boldsymbol{F} \boldsymbol{O}_{\text {pert. }}$.

Observou-se, na amostra gerada, que em média a função objetivo tende a aumentar perante as perturbações. Significa que, em média, não existe risco quando dados de produção, custos ou preços são perturbados. A única exceção que ocorreu foi com as perturbações nas demandas, porém o valor médio caiu muito pouco. Normalmente o projeto é executado uma primeira vez com os valores esperados. As decisões implantadas no $1^{\circ}$ ano de planejamento estão baseadas nestes valores. Como a prática florestal é dinâmica, a cada ano novas decisões tem que ser tomadas, as quais estão baseadas em informações mais atualizadas, eliminando parte do risco devido a falta de informação.

$\mathrm{Na}$ Figura 02 estão representados os resultados das variações sistemáticas de todas as informações trabalhadas. Os limites de variação têm quase a mesma tendência dos resultados das simulações estocásticas. Para o mesmo nível de variação sistemática, as maiores variações 
em $\boldsymbol{F} \boldsymbol{O}_{\text {pert }}$ ocorrem quando são perturbados os preços, depois os custos de manejo, os coeficientes de produção e com quase nenhuma variabilidade, as demandas.

Nas simulações sistemáticas as variações ocorrem em todos os dados, no mesmo sentido, o que não acontece nas simulações estocásticas, quando aleatoriamente os valores são maiores ou menores que a média; por isso os valores de $\boldsymbol{F}_{\boldsymbol{p}}$ ert encontrados tendem a mudar mais, podendo até se tornarem negativos, como no caso da queda dos valores de todos os preços de $50 \% \mathrm{em}$ relação aos valores adotados no modelo básico.

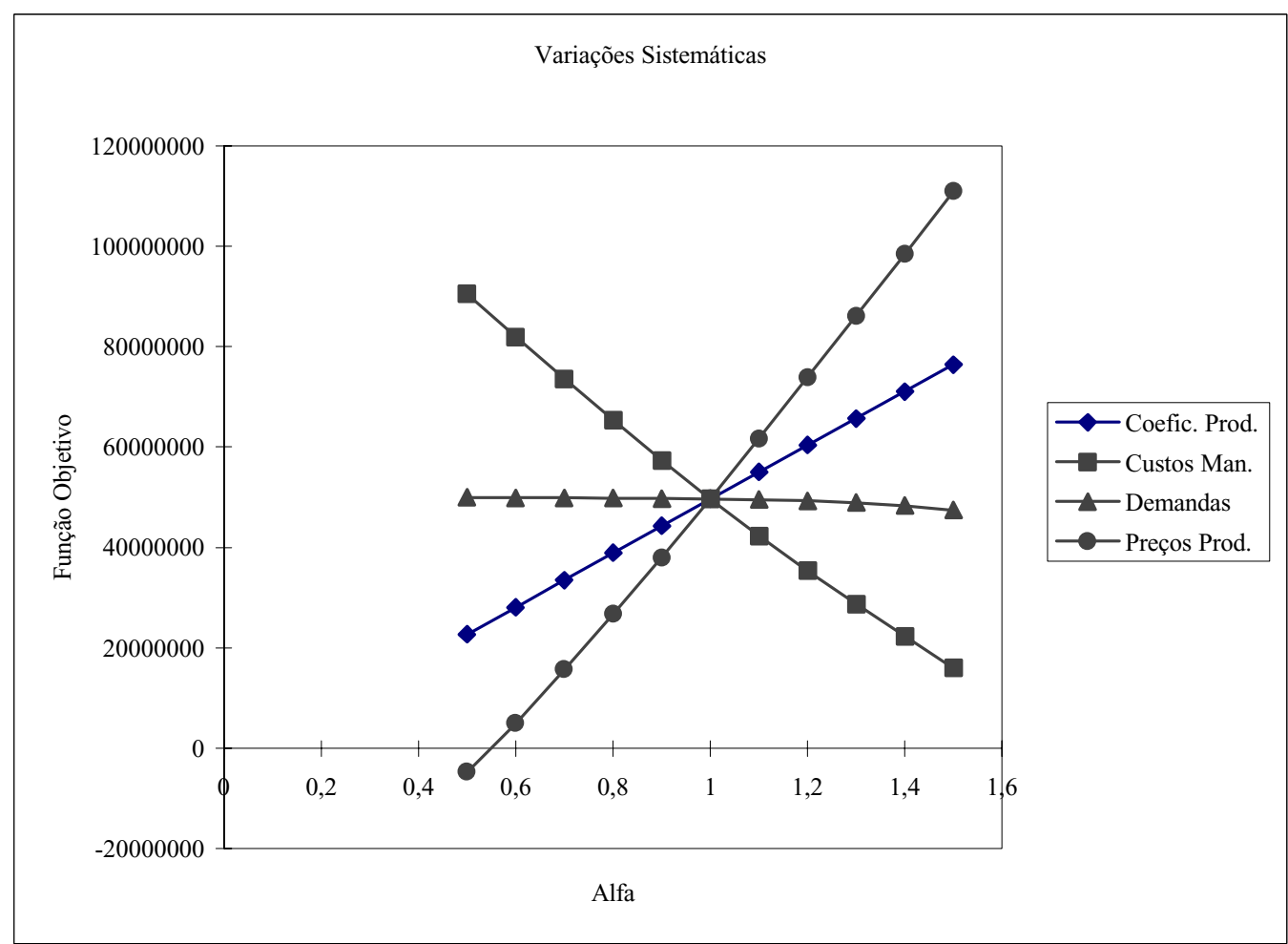

FIGURA 02: EFEITOS DAS VARIAÇÕES SISTEMÁTICAS NA FUNÇÃO OBJETIVO, EM COEFICIENTES DE PRODUÇÃO, CUSTOS DE MANEJO, DEMANDAS E PREÇOS DOS PRODUTOS EM (US\$)

\section{Análise econômica dos coeficientes de variação encontrados}

$\mathrm{Na}$ ausência de um conceito econômico específico para medir as variações da resposta do sistema (valores de $\boldsymbol{F} \boldsymbol{O}_{\text {pert }}$ ), considerou-se razão de variabilidade do coeficiente de produção - sistema como sendo a medida de sensibilidade da resposta do sistema em relação a variações dadas aos coeficientes de produção. Definiu-se este como sendo a razão entre o coeficiente de variação da distribuição da resposta do sistema, o valor de $\boldsymbol{F} \boldsymbol{O}_{\text {pert }}$, e o coeficiente de variação da distribuição de entrada dos coeficientes de produção, conforme a equação

$$
\eta=\frac{C V{ }^{\circ}{ }_{F o} o_{p e r t}}{C V \%_{a_{i j}}}
$$

Este é um conceito pontual, análogo ao conceito econômico de elasticidade ponto. Quanto mais próximo de 0, menos suscetível está o sistema em relação a perturbações daquela variável. Quanto maior $\boldsymbol{\eta}$ maior será a suscetibilidade do sistema ao dado perturbado. 
Da mesma forma, define-se razão de variabilidade do custo de manejo - sistema, razão de variabilidade dos preços dos produtos - sistema e razão de variabilidade das demandas dos produtos - sistema.

Observou-se que o sistema é mais suscetível a variações de preços do que dos outros dados. Esses resultados podem ser vistos nos valores de $\boldsymbol{\eta}$ encontrados para o estudo de caso na Tabela 05.

TABELA 05: VALORES DE $\boldsymbol{\eta}$ - RAZÃO DE VARIABILIDADE DO OBJETO - SISTEMA.

\begin{tabular}{|l|l|l|l|}
\hline Objeto & $\boldsymbol{C} \boldsymbol{V}$ dos dados de entrada & $\boldsymbol{C} \boldsymbol{V}$ dos dados de saída & $\boldsymbol{\eta}$ - razão de variabilidade \\
\hline \multirow{4}{*}{ Coeficientes de Produção } & 10 & 5,4 & 0,54 \\
& 20 & 8,6 & 0,43 \\
& 30 & 13,1 & 0,44 \\
\hline \multirow{4}{*}{ Custos de manejo } & 10 & 3,6 & 0,36 \\
& 20 & 7,2 & 0,36 \\
& 30 & 10,6 & 0,35 \\
\hline \multirow{3}{*}{ Preços dos produtos } & 10 & 13,4 & 1,34 \\
& 20 & 25,9 & 1,30 \\
\hline \multirow{3}{*}{ Demandas dos Produtos } & 20 & 29,4 & 0,98 \\
\hline & 10 & 0,2 & 0,02 \\
& 30 & 0,6 & 0,03 \\
\hline
\end{tabular}

\subsection{CONCLUSÕES}

Sempre houve uma preocupação em relação aos possíveis valores que a função objetivo poderia assumir frente à variabilidade das informações.

No estudo de caso, observou-se que alguns dados interferem mais que outros no valor final da função objetivo (ver Figura 01), informando ao empresário onde ele deve tomar mais cuidado em termos de informação ou onde ele deve buscar a informação adicional, quando possível. Algumas alternativas podem ser consideradas, caso as informações que alimentam o modelo não sejam deterministicamente conhecidas.

Primeiro deve-se examinar as possíveis variabilidades e determinar a sua magnitude, isto é, o que varia e de quanto varia, para depois determinar quais considerações devem ser feitas em relação a essas variabilidades durante o processo de otimização. Se nas análises, as variações dos coeficientes e dados são pequenas ou fazendo pequenas variações nos dados do modelo observa-se que os efeitos na função objetivo, na produção total e escolhas de manejo, são pequenos, pode-se considerar o problema determinístico e usar a média ou a estimativa do valor esperado dos coeficientes, considerando o valor esperado da receita como ótimo. Caso observe-se que a variabilidade só ocorre em $\boldsymbol{c}$ ou em $\boldsymbol{b}$, uma análise paramétrica linear pode ser efetuada.

Se a variação ocorrer em $\boldsymbol{c}$ ou nas restrições ( $\boldsymbol{b}$ e matriz tecnológica $\boldsymbol{A}$ ), o procedimento de usar simulações no modelo de $\boldsymbol{P L}$ mostrou-se adequado para analisar o domínio de variabilidade da função objetivo, pois a distribuição encontrada define os valores possíveis que a função objetivo pode assumir e a probabilidade de ocorrência deles( Ver Tabelas 01 e 02).

Alguns riscos podem ser evitados através de um cuidado melhor na obtenção dos dados, porém alguns dados dependem de informações futuras, tais como preços e mercado futuro e também de ocorrências de catástrofes, como incêndios e pragas, que geram redução nos dados de produção. Pelos resultados da razão de variabilidade (Tabela 05), verificou-se que os preços 
foram os responsáveis pelas maiores variações nos valores de $\boldsymbol{F}_{\text {pert }}$. Na tomada de decisões essa observação é relevante e deve ser considerada quando na análise dos resultados do modelo. Os valores da razão de variabilidade $\boldsymbol{\eta}$ podem ser usados para critério de decisão em subsídios aos incentivos do governo na área florestal. No estudo de caso, mostrou-se que o preço é o melhor incentivo e não o subsídio no custo.

Como um certo grau de risco é inevitável em projetos grandes, estes devem ser planejados à possibilidade de modificações e trocas, isto é, preparar o projeto de uma forma mais flexível possível e depois fazer uso do simulador para se ter uma visão mais ampla das respostas do sistema.

Outra consideração que pode ser feita é que, felizmente, quando um projeto florestal é utilizado ele nunca é executado todo de uma vez e daí tomadas as decisões para todo o horizonte de planejamento. Ele é executado primeiramente com os melhores dados disponíveis para a tomada das primeiras decisões; conforme mais informações são incorporadas ao processo, uma realimentação dos dados no sistema é necessária, reduzindo o risco na informação inicial e na resposta do sistema dada pela distribuição de $\boldsymbol{F} \boldsymbol{O}_{\text {pert }}$.

Agradecimentos: Ao Dr. Luis Roberto Graça (EMBRAPA-PR) e ao Prof. Dr. Anselmo Chaves Neto (UFPR) pelas sugestões da área econômica e estatística, respectivamente, e ao Eng. Romualdo Maestri (PISA Florestal) que forneceu os dados relevantes para esta pesquisa.

\section{REFERÊNCIAS BIBLIOGRÁFICAS}

1. Carnieri,C., Gavinho,L. \& Maestri, R. (1991). Um sistema de planejamento florestal. In: II Encontro de Planejamento Florestal. Anais. Curitiba, 333-346.

2. Clutter, J. \& Fortson, J. \& Pienaar, L. \& Brister, G. \& Bailey, R. (1983) Timber management: A_quantitative approach. Ed. John Wiley \& Sons.

3. Dantzig, G. B. \& Van Slyke R.M. (1967) Generalized Upper Bounding Techniques, Journal of_Computer and System Sciences , 1, 213-226.

4. Filliben, J. (1975). The probability plot correlation coefficient test for normality . Technometrics, 17, n. 1, 111-117.

5. Hof, J. \& Kent, B.M. \& Pickens, J. B. (1992) Chance constraints and chance maximization with random yield coefficients in renewable resource optimization. Forest Science, 38, n.1, 305-323.

6. Hof, J. \& Pickens, J.B. (1991) Chance-constrained and chance-maximizing mathematical programs in renewable resource management. Forest Science. 37 n.1, 308-325.

7. Oliveira, E. B. \& Oliveira, Y. M. M. (1991) SisPinus - Desenvolvimento e perspectivas. In: II Encontro Nacional sobre Planejamento Florestal, Anais, Curitiba, EMBRAPA, 347-360.

8. Pickens, J. \& Dress, P. (1988) Use of stochastic production coefficients in linear programming models: obj. function distribution, feasibility and dual activities, Forest Sc., 34, n.3, 574-591.

9. Volpi, N. M. P. (1997) O impacto de perturbações estocásticas em um modelo de planejamento florestal. Curitiba, . Tese de doutorado. Setor de Ciências Agrárias, UFPR.

10.Wagner, H. (1986) Pesquisa Operacional. 2. ed., Ed. Prentice Hall do Brasil.

11.Weintraub, A. \& Bare, B.B. (1996) New issues in forest land management from an operations research perspective. Interfaces, 26, n. 5, 9-25. 